\title{
Dangerous Disappearing Act: Commensal Gut Microbiota After Acute Severe Insults
}

\author{
Joe Alcock
}

Published online: 23 April 2011

(C) Springer Science+Business Media, LLC (Outside the USA) 2011

The trillions of cells that make up the human gut microbiota are a double-edged sword, often protecting the host from serious infection, but capable of causing lethal inflammation and overwhelming sepsis. This Janus-faced characteristic of the gut microbiota resembles the immune system itself, a powerful defense that can turn on its host. Altered gut microflora is involved in the pathogenesis of a wide variety of diseases, including metabolic syndrome, obesity, congestive heart failure, pancreatitis, inflammatory bowel disease, and critical illness [1-4]. As Hayakawa et al. point out in this issue of Digestive Diseases and Sciences, the gut microbiota is also dramatically changed in acute trauma, out-of-hospital cardiac arrest, and cerebrovascular disease [5]. These authors document the sudden disappearance of commensal bacteria after acute severe insults, raising the question, why?

Most studies of gut microbiota in critical illness have examined hospitalized patients receiving intensive care $[4,6]$. Shimizu et al. have shown that the gut microbiota is significantly altered in patients with systemic inflammatory response syndrome (SIRS) [4, 6]. Until now, the timing of changes in gut ecology has been unknown. In this study, Hayakawa et al. followed 15 acutely critically ill patients from their initial presentation to the emergency department through 2 weeks of hospital care and made three novel

\section{J. Alcock ( ()}

Department of Emergency Medicine, University of New Mexico, MSC10 5560, 1 University of New Mexico, Albuquerque, NM 87131-0001, USA

e-mail: joalcock@salud.unm.edu

\section{J. Alcock}

New Mexico VA Healthcare System,

Emergency Medicine Service, 1501 San Pedro Drive SE, Albuquerque, NM 87108, USA observations [5]. Compared to healthy controls, patients with acute trauma/critical illness had an immediate thousand-fold reduction in the numbers of commensal gut bacteria. Obligate anaerobes were most affected by the sudden destruction of gut flora. Overgrowth of enteric pathogens occurred over the following 2 weeks of hospitalization, while commensal bacteria did not recover.

The cause of altered gut microbiota among hospitalized patients has been variously ascribed to antibiotic treatment, inadequate nutrition, and other stressors [7]. It is well known, for instance, that the obliteration of anaerobic gut flora with broad-spectrum antibiotics predisposes to colonization and overgrowth by Clostridium difficile [8]. Hayakawa et al. showed here that anaerobes and Lactobacillus disappear suddenly and in the early phase of illness. Fecal swabs taken in the emergency room within $6 \mathrm{~h}$ of a patient's arrival showed the destruction of commensal bacteria before antibiotic treatment, suggesting that the mechanism of immediate microbial loss does not involve antibiotics.

Observational studies suggest a link between the preservation of gut microbiota and survival during critical illness [4]. Shimizu et al. reported that gut flora depletion, and loss of obligate anaerobes in particular, increases the risk of gut-derived sepsis, enteritis, and mortality [4, 9]. Changed gut flora and impaired intestinal barrier function are thought to promote the movement of endotoxin and gut bacteria into the portal circulation and gut lymphatics [7]. These events exacerbate system inflammatory damage in hospitalized patients, leading to multi-organ dysfunction syndrome and death [7].

The bacteria that are destroyed during acute severe stress, obligate anaerobes and Lactobacillus, have been shown to protect the host from infection by competitive exclusion of pathogens, production of antimicrobial substances, and prevention of pathogen adhesion to epithelial cells [10]. 
Many anaerobic Bifidobacterium and facultatively anaerobic Lactobacillus species produce bacteriocins that kill enteric pathogens [10, 11]. Lactic acid-producing bacteria also acidify their surroundings, thus, inhibiting the growth of pathogens such as Escherichia coli 0157:H7 [12]. The presence of beneficial commensal bacteria strengthens intestinal barrier function, reduces the translocation of lipopolysaccharide and bacteria, and reverses inflammatory signaling [13]. The vertebrate immune system has evolved the capacity for immunomodulation by specialized commensals [10], resulting in tolerance that protects beneficial bacteria from immune killing and allows some to adhere to intestinal epithelial cells [14].

Hayakawa et al. showed that a dramatic decline of short chain fatty acids (SCFA) coincides with the disappearance of anaerobic gut flora [5]. SCFA are produced during the fermentation of dietary fiber by certain obligate anaerobes and Lactobacillus, and are significantly reduced during critical illness [6]. These organic acids have anti-pathogen activity, including direct toxicity to the cell membranes of some enteric pathogens [15]. SCFA also contribute to the host defense by decreasing intestinal $\mathrm{pH}$, inhibiting pathogen expression of virulence factors, and interfering with the capacity of enteric pathogens to invade intestinal cells [16]. Loss of competitive inhibition by commensal bacteria creates a favorable environment for pathogen growth [10]. It is not surprising, then, that pathogens proliferated after the depletion of commensal bacteria in this study [5].

In light of their protective role, the sudden decline of commensal bacteria and organic acids raises important questions: What causes the destruction of the commensal microbiota? What features of acute severe insults-hypotension, reduced mesenteric blood flow, provision of supplemental oxygen, or systemic inflammation-most affect enteric microorganisms? Why are anaerobic bacteria particularly sensitive to this stress?

The authors propose two possible explanations, hyperoxia and intestinal hypoperfusion, for the destruction of obligate anaerobes. Little is known about the effect of supplemental oxygen on gut flora. However, experiments in mice have shown that hyperoxia improves intestinal barrier function and survival in sepsis [17]. These findings stand in contrast to the impaired barrier function and increased mortality seen in human sepsis following the loss of gut commensals [4]. These opposing results provide circumstantial evidence against a detrimental effect of supplemental oxygen. Hypoperfusion of the gut, an alternative explanation, may also be important in the commensal die-off, but potential mechanisms are unclear.

Another possibility is that the gut flora is actively destroyed and expelled from the host. Human commensals are usually tolerated by the immune system [14], but severe stress may elicit an inflammatory response that results in the loss of protective gut bacteria. If so, then the destruction of human gut flora may resemble the bleaching of corals, in which heat-stressed coral polyps expel symbiotic dinoflagellates called zooxanthellae [18]. After bleaching, corals suffer increased colonization by pathogenic bacteria and die unless the process is reversed [18]. The destruction of human gut microbiota is an equally mysterious process that deserves further study.

Hayakawa et al. report that the enteric pathogens Enterococcus and Pseudomonas show a gradual increase in numbers during the study period [5]. The authors suggest that these changes result, in part, from the removal of competitive inhibition by bacteria that are thought to be beneficial, including Lactobacillus and Bifidobacterium. Host stress is another factor that can promote pathogen growth. Certain enteric pathogens sense and respond to host neuroendocrine stimuli with changes in gene expression, affecting growth and virulence [19].

Exposure to catecholamines, the fight or flight hormones generated during sympathetic autonomic activation, may have contributed to enteric pathogen growth among seriously ill patients in this study. Catecholamines have been shown to induce the growth of enteric pathogens in vitro and in animal models [19]. Hayakawa et al. do not report whether amine vasopressors were administered to the study patients. Amines are often given to treat hypotension in critically ill patients and their yet-unstudied effects on human pathogens in vivo may contribute to the decline in commensal bacteria. More work is needed in order to define the consequences of catecholamines, oxygen, and intravenous fluids on the gut microbiota after acute severe insults.

Hayakawa et al. have identified a promising area of translational research that may lead to changes in the treatment of the acute phase of critical illness. Simple methods such as fecal gram staining [9] have the ability to identify microbiota depletion and can be used in the emergency department. Such diagnostic tests may guide the use of emerging treatments aimed at restoring the gut microbiota. Synbiotics, a combination of beneficial bacteria and growth-supporting nutrients, have been used to treat SIRS [20] and might be most effective when started early. As this field progresses, the gut microbiota will likely be a target of treatment in the prehospital setting and the emergency department, as well as the intensive care unit.

Conflicts of interest There are no financial or other conflicts of interest to disclose.

\section{References}

1. Othman M, Agüero R, Lin HC. Alterations in intestinal microbial flora and human disease. Curr Opin Gastroenterol. 2008;24: $11-16$. 
2. Cani PD, Delzenne NM. Interplay between obesity and associated metabolic disorders: New insights into the gut microbiota. Curr Opin Pharmacol. 2009;9:737-743.

3. Sandek A, Rauchhaus M, Anker SD, von Haehling S. The emerging role of the gut in chronic heart failure. Curr Opin Clin Nutr Metab Care. 2008;11:632-639.

4. Shimizu K, Ogura H, Hamasaki T, Goto M, Tasaki O, Asahara T, Nomoto K, Morotomi M, Matsushima A, Kuwagata Y, Sugimoto H. Altered gut flora are associated with septic complications and death in critically ill patients with systemic inflammatory response syndrome. Dig Dis Sci. 2010;56:1171-1177.

5. Hayakawa M, Asahara T, Henzan N, Murakami H, Yamamoto H, Mukai N, Minami Y, Sugano M, Kubota N, Uegaki S, Kamoshida H, Sawamura A, Nomoto K, Gando S. Dramatic changes of the gut flora immediately after severe and sudden insults. Dig Dis Sci. (Epub ahead of print). doi:10.1007/s10620-011-1649-3

6. Shimizu K, Ogura H, Goto M, Asahara T, Nomoto K, Morotomi M, Yoshiya K, Matsushima A, Sumi Y, Kuwagata Y, Tanaka H, Shimazu T, Sugimoto H. Altered gut flora and environment in patients with severe SIRS. J Trauma. 2006;60:126-133.

7. Iapichino G, Callegari ML, Marzorati S, Cigada M, Corbella D, Ferrari S, Morelli L. Impact of antibiotics on the gut microbiota of critically ill patients. J Med Microbiol. 2008;57:1007-1014.

8. Marra AR, Edmond MB, Wenzel RP, Bearman GM. Hospitalacquired Clostridium difficile-associated disease in the intensive care unit setting: Epidemiology, clinical course and outcome. BMC Infect Dis. 2007;7:42.

9. Shimizu K, Ogura H, Tomono K, Tasaki O, Asahara T, Nomoto K, Morotomi M, Matsushima A, Nakahori Y, Yamano S, Osuka A, Kuwagata Y, Sugimoto H. Patterns of gram-stained fecal flora as a quick diagnostic marker in patients with severe SIRS. Dig Dis Sci. (Epub ahead of print). doi:10.1007/s10620-010-1486-9

10. Walter J, Britton RA, Roos S. Host-microbial symbiosis in the vertebrate gastrointestinal tract and the Lactobacillus reuteri paradigm. Proc Natl Acad Sci USA. 2010;108:4645-4652.
11. Fakhry S, Manzo N, D’Apuzzo E, Pietrini L, Sorrentini I, Ricca E, De Felice M, Baccigalupi L. Characterization of intestinal bacteria tightly bound to the human ileal epithelium. Res Microbiol. 2009;160:817-823.

12. Shin R, Suzuki M, Morishita Y. Influence of intestinal anaerobes and organic acids on the growth of enterohaemorrhagic Escherichia coli O157:H7. J Med Microbiol. 2002;51:201-206.

13. Cani PD, Delzenne NM. The role of the gut microbiota in energy metabolism and metabolic disease. Curr Pharm Des. 2009;15: $1546-1558$.

14. Mowat AM. Anatomical basis of tolerance and immunity to intestinal antigens. Nat Rev Immunol. 2003;3:331-341.

15. Lawhon SD, Maurer R, Suyemoto M, Altier C. Intestinal shortchain fatty acids alter Salmonella typhimurium invasion gene expression and virulence through BarA/SirA. Mol Microbiol. 2002;46:1451-1464.

16. Lin CK, Tsai HC, Lin PP, Tsen HY, Tsai CC. Lactobacillus acidophilus LAP5 able to inhibit the Salmonella choleraesuis invasion to the human Caco-2 epithelial cell. Anaerobe. 2008;14: 251-255.

17. Gennari R, Alexander JW. Effects of hyperoxia on bacterial translocation and mortality during gut-derived sepsis. Arch Surg. 1996;131:57-62.

18. Rosenberg E, Koren O, Reshef L, Efrony R, Zilber-Rosenberg I. The role of microorganisms in coral health, disease and evolution. Nat Rev Microbiol. 2007;5:355-362.

19. Freestone PP, Sandrini SM, Haigh RD, Lyte M. Microbial endocrinology: How stress influences susceptibility to infection. Trends Microbiol. 2008;16:55-64.

20. Shimizu K, Ogura H, Goto M, Asahara T, Nomoto K, Morotomi M, Matsushima A, Tasaki O, Fujita K, Hosotsubo H, Kuwagata Y, Tanaka H, Shimazu T, Sugimoto H. Synbiotics decrease the incidence of septic complications in patients with severe SIRS: A preliminary report. Dig Dis Sci. 2009;54:1071-1078. 